\title{
Solve the split equality problem by a projection algorithm with inertial effects
}

\author{
Qiao-Li Dong*, Dan Jiang \\ Tianjin Key Laboratory for Advanced Signal Processing, College of Science, Civil Aviation University of China, Tianjin 300300, China.
}

Communicated by Y. H. Yao

\begin{abstract}
The split equality problem has wide applicability in many fields of applied mathematics. In this paper, by using the inertial extrapolation, we introduce an inertial projection algorithm for solving the split equality problem. The weak convergence of the proposed algorithm is shown. Finally, we present a numerical example to illustrate the efficiency of the inertial projection algorithm. (c) 2017 All rights reserved.
\end{abstract}

Keywords: Split equality problem, projection algorithm, inertial extrapolation. 2010 MSC: 47H05, 47H07, 47H10.

\section{Introduction}

In this article, we shall consider the split equality problem (SEP) which was firstly introduced by Moudafi and Oliny [18].

Problem 1.1. Find $x, y$ with the property

$$
x \in C, y \in Q \text {, such that } A x=B y,
$$

where $\mathrm{C} \subset \mathrm{H}_{1}, \mathrm{Q} \subset \mathrm{H}_{2}$ are two nonempty closed convex sets, $\mathrm{A}: \mathrm{H}_{1} \rightarrow \mathrm{H}_{3}, \mathrm{~B}: \mathrm{H}_{2} \rightarrow \mathrm{H}_{3}$ are two bounded linear operators, and $\mathrm{H}_{1}, \mathrm{H}_{2}$ and $\mathrm{H}_{3}$ are real Hilbert spaces.

It is obvious that the SEP allows asymmetric and partial relations between the variables $x$ and $y$. Many problems in mathematics and other sciences can be regarded as a split equality problem, such as the variational form of a PDE's in domain decomposition for PDE's [3], the agents who interplay only via some components of their decision variables in decision [2] and the weak coupling between the vector of doses absorbed in all voxels and that of the radiation intensity in the (IMRT) [7].

Many methods for computing the solution of Problem 1.1 are projection methods, which have been extensively studied in the literature [10, 15, 16, 19]. Byrne and Moudafi [6] introduced the classical

\footnotetext{
${ }^{*}$ Corresponding author

Email addresses: dongql@lsec.cc.ac.cn (Qiao-Li Dong), danjiangmath@163.com (Dan Jiang)
} 
projection gradient algorithm, which is also called as the simultaneous iterative methods [17]:

$$
\left\{\begin{array}{l}
x_{k+1}=P_{C}\left(x_{k}-\gamma_{k} A^{*}\left(A x_{k}-B y_{k}\right)\right) \\
y_{k+1}=P_{Q}\left(y_{k}+\gamma_{k} B^{*}\left(A x_{k}-B y_{k}\right)\right)
\end{array}\right.
$$

where $\gamma_{k} \in\left(\varepsilon, 2 /\left(\lambda_{A}+\lambda_{B}\right)-\varepsilon\right), \lambda_{A}$ and $\lambda_{B}$ are the operator (matrix) norms $\|A\|$ and $\|B\|$ (or the largest eigenvalues of $A^{*} A$ and $B * B$ ), respectively. To determine stepsize $\gamma_{k}$, one needs first calculate (or estimate) the operator norms $\|A\|$ and $\|B\|$. In general, it is difficult or even impossible.

In order to overcome this, the authors [11] proposed a choice of the stepsize $\gamma_{k}$ for the projection algorithm (1.1) as follows:

Algorithm 1.2.

$$
\gamma_{k}=\sigma_{k} \min \left\{\frac{\left\|A \bar{x}_{k}-B \bar{y}_{k}\right\|^{2}}{\left\|A^{*}\left(A \bar{x}_{k}-B \bar{y}_{k}\right)\right\|^{2}}, \frac{\left\|A \bar{x}_{k}-B \bar{y}_{k}\right\|^{2}}{\left\|B^{*}\left(A \bar{x}_{k}-B \bar{y}_{k}\right)\right\|^{2}}\right\},
$$

where $0<\sigma_{k}<1$. Note that the choice of the stepsize $\gamma_{k}$ in (1.2) is independent of the norms $\|A\|$ and $\|\mathrm{B}\|$.

As an acceleration process, the inertial extrapolation algorithms were widely studied. The researchers constructed many iterative algorithms by using inertial extrapolation, such as inertial forward-backward algorithm [14], inertial extragradient method $[9,12,13]$ and fast iterative shrinkage thresholding algorithm (FISTA) $([5,8])$. The main feature of the inertial extrapolation algorithms is that the next iterate is defined by making use of the previous two iterates.

In this paper, by using the inertial extrapolation, we introduce an inertial projection algorithm (1.1) as follows:

\section{Algorithm 1.3.}

$$
\left\{\begin{array}{l}
\left(\bar{x}_{k}, \bar{y}_{k}\right)=\left(x_{k}, y_{k}\right)+\alpha_{k}\left(x_{k}-x_{k-1}, y_{k}-y_{k-1}\right), \\
x_{k+1}=P_{C}\left(\bar{x}_{k}-\gamma_{k} A^{*}\left(A \bar{x}_{k}-B \bar{y}_{k}\right)\right), \\
y_{k+1}=P_{Q}\left(\bar{y}_{k}+\gamma_{k} B^{*}\left(A \bar{x}_{k}-B \bar{y}_{k}\right)\right),
\end{array}\right.
$$

where $\alpha_{k} \in(0,1)$ and the stepsize $\gamma_{k}$ is chosen in the same way as (1.2).

The structure of the paper is as follows. In the next section, we present some lemmas which will be used in the main results. In Section 3, the weak convergence theorem of the inertial projection algorithm is given. In the final section, Section 4, some numerical results are provided, which show the advantages of the proposed algorithm.

\section{Preliminaries}

In this section, we present some lemmas which will be used in the proof of the main results.

Definition 2.1. Let $\mathrm{K}$ be a closed convex subset of a real Hilbert space $\mathrm{H}$. $\mathrm{P}_{\mathrm{K}}$ is called the projection from $\mathrm{H}$ on $\mathrm{K}$, if for each $x \in H, \mathrm{P}_{\mathrm{K}} x$ is the only point in $\mathrm{K}$ such that $\left\|x-\mathrm{P}_{\mathrm{K}} x\right\|=\inf \{\|x-z\|: z \in \mathrm{K}\}$.

The following lemma is a useful characterization of projections.

Lemma 2.2. Let $\mathrm{K}$ be a closed convex subset of a real Hilbert space $\mathrm{H}$. Given $\mathrm{x} \in \mathrm{H}$ and $z \in \mathrm{K}$. Then $z=\mathrm{P}_{\mathrm{K}} \mathrm{x}$, if and only if there holds the relation:

$$
\langle x-z, y-z\rangle \leqslant 0, \quad \forall y \in K .
$$

Lemma 2.3. For any $x, y \in \mathrm{H}$ and $z \in \Omega$, it holds

$$
\left\|\mathrm{P}_{\Omega}(x)-z\right\|^{2} \leqslant\|x-z\|^{2}-\left\|\mathrm{P}_{\Omega}(x)-x\right\|^{2} .
$$


Lemma 2.4 ([1, Lemma 3]). Let $\left(\psi_{n}\right),\left(\delta_{n}\right)$ and $\left(\alpha_{n}\right)$ be sequences in $[0,+\infty)$ such that

$$
\psi_{n+1} \leqslant \psi_{n}+\alpha_{n}\left(\psi_{n}-\psi_{n-1}\right)+\delta_{n},
$$

for all $n \geqslant 1, \sum_{n=1}^{\infty} \delta_{n}<+\infty$ and there exists a real number $\alpha$ with $0 \leqslant \alpha_{n} \leqslant \alpha<1$, for all $n \in \mathbb{N}$. Then the following hold:

(i) $\sum_{n \geqslant 1}\left[\psi_{n}-\psi_{n-1}\right]_{+}<+\infty$, where $[t]_{+}=\max \{t, 0\}$;

(ii) there exists $\psi^{*} \in[0,+\infty)$ such that $\lim _{n \rightarrow+\infty} \psi_{n}=\psi^{*}$.

Finally, we recall a well-known result on weak convergence in Hilbert spaces.

Lemma 2.5 ([4]). Let $\mathrm{H}$ be a Hilbert space and $\|\cdot\|$ be a norm on $\mathrm{H}$, then,

$$
\|\mathrm{tx}+(1-\mathrm{t}) \mathrm{y}\|^{2}=\mathrm{t}\|x\|^{2}+(1-\mathrm{t})\|\mathrm{y}\|^{2}-\mathrm{t}(1-\mathrm{t})\|x-\mathrm{y}\|^{2}, \quad \forall \mathrm{t} \in \mathrm{R}, \forall x, y \in H .
$$

\section{The main results}

In this section, we present the weak convergence theorem and its proof for the inertial projection algorithm (1.3).

We make the following assumptions to $\left\{\alpha_{k}\right\}$ :

(C1) $0 \leqslant \alpha_{k} \leqslant \alpha$, where $\alpha \in[0,1)$;

(C2) $\sum_{k=1}^{+\infty} \alpha_{k}\left(\left\|x_{k}-x_{k-1}\right\|^{2}+\left\|y_{k}-y_{k-1}\right\|^{2}\right)<+\infty$;

(C3) $\lim _{k \rightarrow \infty} \alpha_{k}\left(\left\|x_{k}-x_{k-1}\right\|+\left\|y_{k}-y_{k-1}\right\|\right)=0$.

\section{Remark 3.1.}

(1) Comparing other works [8, 16], we need the additional assumptions (C3) to present the convergence theorem.

(2) According to Moudafi's comments in [16], assumptions (C2) and (C3) involve the iterates that are a priori unknown. In practice, it is easy to enforce it by applying an appropriate on-line rule. For example, choosing

$$
\alpha_{k}=\min \left(\frac{1}{(k+1)^{4} \eta_{k}}, \frac{1}{(k+1) \zeta_{k}}, 0.94\right),
$$

where

$$
\eta_{k}=\left\|x_{k}-x_{k-1}\right\|^{2}+\left\|y_{k}-y_{k-1}\right\|^{2}, \zeta_{k}=\left\|x_{k}-x_{k-1}\right\|+\left\|y_{k}-y_{k-1}\right\| .
$$

Theorem 3.2. Let the sequence $\left(x_{k}, y_{k}\right)$ be generated by Algorithm 1.3. Suppose the assumptions (C1) and (C2) hold. Then $\left(\left\|x_{k}-x^{*}\right\|^{2}+\left\|y_{k}-y^{*}\right\|^{2}\right)$ is convergent with $\left(x^{*}, y^{*}\right) \in \Gamma$.

Proof. Take $\left(x^{*}, y^{*}\right) \in \Gamma$, i.e., $x^{*} \in C, y^{*} \in Q, A x^{*}=B y^{*}$. From Lemma 2.3, the second equality of (1.3) successively gives

$$
\begin{aligned}
\left\|x_{k+1}-x^{*}\right\|^{2} & =\left\|P_{C}\left(\bar{x}_{k}-\gamma_{k} A^{*}\left(A \bar{x}_{k}-B \bar{y}_{k}\right)-x^{*}\right)\right\|^{2} \\
& \leqslant\left\|\bar{x}_{k}-\gamma_{k} A^{*}\left(A \bar{x}_{k}-B \bar{y}_{k}\right)-x^{*}\right\|^{2} \\
& \leqslant\left\|\bar{x}_{k}-x^{*}\right\|^{2}+\gamma_{k}^{2}\left\|A^{*}\left(A \bar{x}_{k}-B \bar{y}_{k}\right)\right\|^{2}-2 \gamma_{k}\left\langle A \bar{x}_{k}-A x^{*}, A \bar{x}_{k}-B \bar{y}_{k}\right\rangle .
\end{aligned}
$$

Using the equality

$$
-2\left\langle A \bar{x}_{k}-A x^{*}, A \bar{x}_{k}-B \bar{y}_{k}\right\rangle=-\left\|A \bar{x}_{k}-B \bar{y}_{k}\right\|^{2}-\left\|A \bar{x}_{k}-A x^{*}\right\|^{2}+\left\|B \bar{y}_{k}-A x^{*}\right\|^{2},
$$


we obtain

$$
\begin{aligned}
\left\|x_{k+1}-x^{*}\right\|^{2} \leqslant & \left\|\bar{x}_{k}-x^{*}\right\|^{2}+\gamma_{k}^{2}\left\|A^{*}\left(A \bar{x}_{k}-B \bar{y}_{k}\right)\right\|^{2}-\gamma_{k}\left\|A \bar{x}_{k}-B \bar{y}_{k}\right\|^{2} \\
& -\gamma_{k}\left\|A \bar{x}_{k}-A x^{*}\right\|^{2}+\gamma_{k}\left\|B \bar{y}_{k}-A x^{*}\right\|^{2} .
\end{aligned}
$$

Similarly, the third equality of (1.3) leads to

$$
\begin{gathered}
\left\|y_{k+1}-y^{*}\right\|^{2} \leqslant \\
-\bar{y}_{k}-y^{*}\left\|^{2}+\gamma_{k}^{2}\right\| B^{*}\left(A \bar{x}_{k}-B \bar{y}_{k}\right)\left\|^{2}-\gamma_{k}\right\| A \bar{x}_{k}-B \bar{y}_{k} \|^{2} \\
-\gamma_{k}\left\|B \bar{y}_{k}-B y^{*}\right\|^{2}+\gamma_{k}\left\|A \bar{x}_{k}-B y^{*}\right\|^{2} .
\end{gathered}
$$

By adding the two last inequalities and taking into account the fact that $A x^{*}=B y^{*}$, we finally get

$$
\begin{aligned}
\left\|x_{k+1}-x^{*}\right\|^{2}+\left\|y_{k+1}-y^{*}\right\|^{2} \leqslant & \left\|\bar{x}_{k}-x^{*}\right\|^{2}+\left\|\bar{y}_{k}-y^{*}\right\|^{2}-\gamma_{k}\left(\left\|A \bar{x}_{k}-B \bar{y}_{k}\right\|^{2}-\gamma_{k}\left\|A^{*}\left(A \bar{x}_{k}-B \bar{y}_{k}\right)\right\|^{2}\right) \\
& -\gamma_{k}\left(\left\|A \bar{x}_{k}-B \bar{y}_{k}\right\|^{2}-\gamma_{k}\left\|B^{*}\left(A \bar{x}_{k}-B \bar{y}_{k}\right)\right\|^{2}\right) .
\end{aligned}
$$

The first equality of (1.3) and Lemma 2.5 imply

$$
\begin{aligned}
\left\|\bar{x}_{k}-x^{*}\right\|^{2} & =\left\|\left(1+\alpha_{k}\right)\left(x_{k}-x^{*}\right)-\alpha_{k}\left(x_{k-1}-x^{*}\right)\right\|^{2} \\
& =\left(1+\alpha_{k}\right)\left\|x_{k}-x^{*}\right\|^{2}-\alpha_{k}\left\|x_{k-1}-x^{*}\right\|^{2}+\alpha_{k}\left(1+\alpha_{k}\right)\left\|x_{k}-x_{k-1}\right\|^{2} .
\end{aligned}
$$

Similarly, we obtain

$$
\left\|\bar{y}_{k}-y^{*}\right\|^{2}=\left(1+\alpha_{k}\right)\left\|y_{k}-y^{*}\right\|^{2}-\alpha_{k}\left\|y_{k-1}-y^{*}\right\|^{2}+\alpha_{k}\left(1+\alpha_{k}\right)\left\|y_{k}-y_{k-1}\right\|^{2} .
$$

Set $\varphi_{k}:=\left\|x_{k}-x^{*}\right\|^{2}+\left\|y_{k}-y^{*}\right\|^{2}$. Combining (3.1), (3.2), (3.3), we obtain

$$
\begin{aligned}
\varphi_{k+1}-\varphi_{k} \leqslant & \alpha_{k}\left(\varphi_{k}-\varphi_{k-1}\right)-\gamma_{k}\left(\left\|A \bar{x}_{k}-B \bar{y}_{k}\right\|^{2}-\gamma_{k}\left\|A^{*}\left(A \bar{x}_{k}-B \bar{y}_{k}\right)\right\|^{2}\right) \\
& -\gamma_{k}\left(\left\|A \bar{x}_{k}-B \bar{y}_{k}\right\|^{2}-\gamma_{k}\left\|B^{*}\left(A \bar{x}_{k}-B \bar{y}_{k}\right)\right\|^{2}\right) \\
& +\alpha_{k}\left(1+\alpha_{k}\right)\left[\left\|x_{k}-x_{k-1}\right\|^{2}+\left\|y_{k}-y_{k-1}\right\|^{2}\right] \\
\leqslant & \alpha_{k}\left(\varphi_{k}-\varphi_{k-1}\right)+\delta_{k}
\end{aligned}
$$

where $\delta_{k}:=\alpha_{k}\left(1+\alpha_{k}\right)\left[\left\|x_{k}-x_{k-1}\right\|^{2}+\left\|y_{k}-y_{k-1}\right\|^{2}\right]$. By using (C2) and Lemma 2.4, we obtain that $\varphi_{k}$ consequently converges to some finite limit, say $\varphi\left(x^{*}, y^{*}\right)$.

Theorem 3.3. Let the sequence $\left(x_{k}, y_{k}\right)$ be generated by Algorithm 1.3. Assume $\sigma_{k} \in[\varepsilon, 1-\varepsilon], \varepsilon \in(0,1 / 2]$ and suppose the assumptions (C1)-(C3) hold. Then

$$
\lim _{k \rightarrow \infty}\left\|A x_{k}-B y_{k}\right\|=\lim _{k \rightarrow \infty}\left\|A \bar{x}_{k}-B \bar{y}_{k}\right\|=0 .
$$

Proof. Now, we divide the proof of the first conclusion into two parts.

Case 1. Suppose that there exists $k_{0}$ such that $\left\|A^{*}\left(A \bar{x}_{k}-B \bar{y}_{k}\right)\right\| \geqslant\left\|B^{*}\left(A \bar{x}_{k}-B \bar{y}_{k}\right)\right\|$, for all $k \geqslant k_{0}$. In this situation, $\gamma_{k}=\sigma_{k} \frac{\left\|A \bar{x}_{k}-B \bar{y}_{k}\right\|^{2}}{\left\|A^{*}\left(A \bar{x}_{k}-B \bar{y}_{k}\right)\right\|^{2}}$. Using (3.4) and Theorem 3.2, we obtain

$$
\lim _{k \rightarrow \infty} \gamma_{k}\left(\left\|A \bar{x}_{k}-B \bar{y}_{k}\right\|^{2}-\gamma_{k}\left\|A^{*}\left(A \bar{x}_{k}-B \bar{y}_{k}\right)\right\|^{2}\right)=\lim _{k \rightarrow \infty} \sigma_{k}\left(1-\sigma_{k}\right) \frac{\left\|A \bar{x}_{k}-B \bar{y}_{k}\right\|^{4}}{\left\|A^{*}\left(A \bar{x}_{k}-B \bar{y}_{k}\right)\right\|^{2}}=0,
$$

which together with $\sigma_{k} \in[\varepsilon, 1-\varepsilon]$ implies

$$
\lim _{k \rightarrow \infty} \frac{\left\|A \bar{x}_{k}-B \bar{y}_{k}\right\|^{2}}{\left\|A^{*}\left(A \bar{x}_{k}-B \bar{y}_{k}\right)\right\|}=0
$$

Using $\gamma_{k}\left\|A^{*}\left(A \bar{x}_{k}-B \bar{y}_{k}\right)\right\|=\sigma_{k} \frac{\left\|A \bar{x}_{k}-B \bar{y}_{k}\right\|^{2}}{\left\|A^{*}\left(A \bar{x}_{k}-B \bar{y}_{k}\right)\right\|}$, we get

$$
\lim _{k \rightarrow \infty} \gamma_{k}\left\|A^{*}\left(A \bar{x}_{k}-B \bar{y}_{k}\right)\right\|=0 \text {. }
$$


From the assumption $\left\|A^{*}\left(A \bar{x}_{k}-B \bar{y}_{k}\right)\right\| \geqslant\left\|B^{*}\left(A \bar{x}_{k}-B \bar{y}_{k}\right)\right\|$, it follows

$$
\lim _{k \rightarrow \infty} \gamma_{k}\left\|B^{*}\left(A \bar{x}_{k}-B \bar{y}_{k}\right)\right\|=0
$$

It is easy to show that

$$
\left\|A \bar{x}_{k}-B \bar{y}_{k}\right\| \leqslant\|A\| \frac{\left\|A \bar{x}_{k}-B \bar{y}_{k}\right\|^{2}}{\left\|A *\left(A \bar{x}_{k}-B \bar{y}_{k}\right)\right\|}
$$

Consequently, we get

$$
\lim _{k \rightarrow \infty}\left\|A \bar{x}_{k}-B \bar{y}_{k}\right\|=0 .
$$

Conversely, suppose that there exists $k_{1}$ such that $\left\|A^{*}\left(A \bar{x}_{k}-B \bar{y}_{k}\right)\right\| \leqslant\left\|B^{*}\left(A \bar{x}_{k}-B \bar{y}_{k}\right)\right\|$, for all $k \geqslant k_{1}$, following the above process, we obtain the results.

Case 2. Suppose that there does not exist $k_{0}$ such that $\left\|A^{*}\left(A \bar{x}_{k}-B \bar{y}_{k}\right)\right\| \geqslant\left\|B^{*}\left(A \bar{x}_{k}-B \bar{y}_{k}\right)\right\|$ or $\| A^{*}\left(A \bar{x}_{k}-\right.$ $\left.B \bar{y}_{k}\right)\|\leqslant\| B^{*}\left(A \bar{x}_{k}-B \bar{y}_{k}\right) \|$, for all $k \geqslant k_{0}$. We divide the sequence $\left\|A^{*}\left(A \bar{x}_{k}-B \bar{y}_{k}\right)\right\|$ into two subsequences: one subsequence satisfies $\left\|A^{*}\left(A \bar{x}_{k}-B \bar{y}_{k}\right)\right\| \geqslant\left\|B^{*}\left(A x_{k}-B y_{k}\right)\right\|$ denoted by $A^{*}\left(A x_{k_{m}}-B y_{k_{m}}\right)$ and the other subsequence satisfies $\left\|A^{*}\left(A \bar{x}_{k}-B \bar{y}_{k}\right)\right\|<\left\|B^{*}\left(A \bar{x}_{k}-B \bar{y}_{k}\right)\right\|$, denoted by $A^{*}\left(A \bar{x}_{k_{n}}-B \bar{y}_{k_{n}}\right)$. Following the process of Case 1, we show that the results hold for the subsequences with $k_{m}$ and $k_{n}$. Hence we obtain $\lim _{k \rightarrow \infty}\left\|A \bar{x}_{k}-B \bar{y}_{k}\right\|=0$.

Next we show $\lim _{k \rightarrow \infty}\left\|A x_{k}-B y_{k}\right\|=0$. From the linearity of the operators $A$ and $B$, and the first equality of (1.3), we obtain

$$
\begin{aligned}
\left\|A x_{k}-B y_{k}\right\| & =\left\|\left[A \bar{x}_{k}-\alpha_{k} A\left(x_{k}-x_{k-1}\right)\right]-\left[B \bar{y}_{k}-\alpha_{k} B\left(y_{k}-y_{k-1}\right)\right]\right\| \\
& \leqslant\left\|A \bar{x}_{k}-B \bar{y}_{k}\right\|+\alpha_{k}\left(\left\|A\left(x_{k}-x_{k-1}\right)\right\|+\alpha_{k}\left\|B\left(y_{k}-y_{k-1}\right)\right\|\right) \\
& \leqslant\left\|A \bar{x}_{k}-B \bar{y}_{k}\right\|+(\|A\|+\|B\|) \alpha_{k}\left(\left\|x_{k}-x_{k-1}\right\|+\left\|y_{k}-y_{k-1}\right\|\right),
\end{aligned}
$$

which with $\lim _{k \rightarrow \infty}\left\|A \bar{x}_{k}-B \bar{y}_{k}\right\|=0$ and (C3) implies

$$
\lim _{k \rightarrow \infty}\left\|A x_{k}-B y_{k}\right\|=0
$$

Theorem 3.4. Assume $\sigma_{k} \in[\varepsilon, 1-\varepsilon], \varepsilon \in(0,1 / 2]$ and suppose the assumptions (C1)-(C3) hold. Then the sequence $\left(x_{k}, y_{k}\right)$ generated by Algorithm 1.3 weakly converges to a solution of the SEP. Furthermore, both $\left(x_{k}\right)$ and $\left(y_{k}\right)$ are asymptotically regular.

Proof. We first prove that $\left(x_{k}\right)$ and $\left(y_{k}\right)$ are asymptotically regular. Indeed, the first and the second equalities of (1.3) give

$$
\begin{aligned}
\left\|x_{k+1}-x_{k}\right\| & =\left\|P_{C}\left(\bar{x}_{k}-\gamma_{k} A^{*}\left(A \bar{x}_{k}-B \bar{y}_{k}\right)\right)-x_{k}\right\| \\
& \leqslant\left\|\bar{x}_{k}-\gamma_{k} A^{*}\left(A \bar{x}_{k}-B \bar{y}_{k}\right)-x_{k}\right\| \\
& \leqslant\left\|\alpha_{k}\left(x_{k}-x_{k-1}\right)-\gamma_{k} A^{*}\left(A \bar{x}_{k}-B \bar{y}_{k}\right)\right\| \\
& \leqslant \alpha_{k}\left\|x_{k}-x_{k-1}\right\|+\gamma_{k}\left\|A^{*}\left(A \bar{x}_{k}-B \bar{y}_{k}\right)\right\| \\
& \leqslant \alpha_{k}\left\|x_{k}-x_{k-1}\right\|+\gamma_{k}\|A\|\left\|A \bar{x}_{k}-B \bar{y}_{k}\right\| .
\end{aligned}
$$

Using (C3) and Theorem 3.3, we have

$$
\lim _{k \rightarrow \infty}\left\|x_{k+1}-x_{k}\right\|=0
$$

Similarly, we get

$$
\lim _{k \rightarrow \infty}\left\|y_{k+1}-y_{k}\right\|=0
$$


So, $\left(x_{k}\right)$ and $\left(y_{k}\right)$ are asymptotically regular.

The first equality of (1.3) gives

$$
\begin{aligned}
\left\|\bar{x}_{k}-x_{k+1}\right\| & =\left\|x_{k}-x_{k+1}+\alpha_{k}\left(x_{k}-x_{k-1}\right)\right\| \\
& \leqslant\left\|x_{k}-x_{k+1}\right\|+\alpha_{k}\left\|x_{k}-x_{k-1}\right\|,
\end{aligned}
$$

which with (C3) and (3.5) yields

$$
\lim _{k \rightarrow \infty}\left\|\bar{x}_{k}-x_{k+1}\right\|=0 .
$$

Similarly, we have

$$
\lim _{k \rightarrow \infty}\left\|\bar{y}_{k}-y_{k+1}\right\|=0
$$

From (1.2), we get

$$
\gamma_{k} \geqslant \varepsilon \min \left\{\frac{1}{\|A\|^{2}}, \frac{1}{\|B\|^{2}}\right\}
$$

So, it follows

$$
\lim _{k \rightarrow \infty} \frac{\bar{x}_{k}-x_{k+1}}{\gamma_{k}}=0, \text { and } \quad \lim _{k \rightarrow \infty} \frac{\bar{y}_{k}-y_{k+1}}{\gamma_{k}}=0 .
$$

Let $(\hat{x}, \hat{y}) \in \omega_{w}\left(x_{k}, y_{k}\right)$, then there exists a subsequence of $\left(x_{k}\right)$ (resp. $\left.\left(y_{k}\right)\right)$ (again labeled ( $\left.x_{k}\right)$ (resp. $\left.\left.\left(y_{k}\right)\right)\right)$ which converges weakly to $\hat{x}$ (resp. $\left.\hat{y}\right)$. The two equalities in (1.3) can be rewritten as:

$$
\left\{\begin{array}{l}
\frac{\bar{x}_{k}-x_{k+1}}{\gamma_{k}}-A^{*}\left(A \bar{x}_{k}-B \bar{y}_{k}\right) \in N_{C}\left(x_{k+1}\right), \\
\frac{\bar{y}_{k}-y_{k+1}}{\gamma_{k}}-B^{*}\left(A \bar{x}_{k}-B \bar{y}_{k}\right) \in N_{Q}\left(y_{k+1}\right) .
\end{array}\right.
$$

Since the graphs of the maximal monotone operators $N_{C}, N_{Q}$ are weakly-strongly closed, by passing to the limit in the last inclusions and using Theorem 3.3, we obtain $0 \in N_{C}(\hat{x})$ and $0 \in N_{Q}(\hat{y})$ which are equivalent to

$$
\hat{x} \in C \text { and } \hat{y} \in Q \text {. }
$$

Furthermore, the weak convergence of $\left(A x_{k}-B y_{k}\right)$ to $A \hat{x}-B \hat{y}$ and lower semicontinuity of the norm imply

$$
\|A \hat{x}-B \hat{y}\| \leqslant \liminf _{k \rightarrow \infty}\left\|A x_{k}-B y_{k}\right\|=0,
$$

where the equality comes from Theorem 3.3. Hence, $(\hat{x}, \hat{y}) \in \Gamma$.

To show the uniqueness of the weak cluster points, we will use the same trick as in the celebrated Opial Lemma. Indeed, let $(\bar{x}, \bar{y})$ be other weak cluster point of $\left(x_{k}, y_{k}\right)$. By passing to the limit in the relation

$$
\varphi_{k}(\hat{x}, \hat{y})=\varphi_{k}(\bar{x}, \bar{y})+\|\hat{x}-\bar{x}\|^{2}+\|\hat{y}-\bar{y}\|^{2}+2\left\langle x_{k}-\bar{x}, \bar{x}-\hat{x}\right\rangle+2\left\langle y_{k}-\bar{y}, \bar{y}-\hat{y}\right\rangle,
$$

we obtain

$$
\varphi(\hat{x}, \hat{y})=\varphi(\bar{x}, \bar{y})+\|\hat{x}-\bar{x}\|^{2}+\|\hat{y}-\bar{y}\|^{2} .
$$

Reversing the role of $(\hat{x}, \hat{y})$ and $(\bar{x}, \bar{y})$, we also have

$$
\varphi(\bar{x}, \bar{y})=\varphi(\hat{x}, \hat{y})+\|\hat{x}-\bar{x}\|^{2}+\|\hat{y}-\bar{y}\|^{2} .
$$

By adding the two last equalities, we obtain

$$
\|\hat{x}-\bar{x}\|^{2}+\|\hat{y}-\bar{y}\|^{2}=0 .
$$

Hence, $(\hat{x}, \hat{y})=(\bar{x}, \bar{y})$. This implies that the whole sequence $\left(x_{k}, y_{k}\right)$ weakly converges to a solution of the $\mathrm{SEP}$, which completes the proof. 


\section{Preliminary numerical results}

In this section, we consider a numerical example in [11] to demonstrate the effectiveness of Algorithm 1.3. We apply Algorithm 1.3 to solve a numerical example, and compare the numerical results with those of Algorithm 1.2.

We denote the vector with all elements 0 by $e_{0}$, and the vector with all elements 1 by $e_{1}$ in what follows. In the numerical results listed in the following table, 'Iter.' and 'Sec.' denote the number of iterations and the cpu time in seconds, respectively.

Example 4.1. The SEP with $A=\left(a_{i j}\right)_{J \times N}, B=\left(b_{i j}\right)_{J \times M}, C=\left\{x \in \mathbb{R}^{N} \mid\|x\| \leqslant 0.25\right\}, Q=\left\{y \in \mathbb{R}^{M} \mid e_{0} \leqslant\right.$ $y \leqslant u\}$, where $a_{i j} \in[0,1], b_{i j} \in[0,1]$ and $u \in\left[e_{1}, 2 e_{1}\right]$ are all generated randomly. In the implementations, we take $\|A x-B y\|<\varepsilon=10^{-4}$ as the stopping criterion. Take the initial value $x_{0}=10 e_{1}, y_{0}=-10 e_{1}$ for two algorithms.

We make comparison of Algorithm 1.3 with Algorithm 1.2 with different $J, N, M$, and report the results in Table 1. We take $\sigma_{k}=0.69, \gamma_{k}=0.69 \times \min \left\{\frac{\left\|A \bar{x}_{k}-B \bar{y}_{k}\right\|^{2}}{\left\|A^{*}\left(A \bar{x}_{k}-B \bar{y}_{k}\right)\right\|^{2}}, \frac{\left\|A \bar{x}_{k}-B \bar{y}_{k}\right\|^{2}}{\left\|B B^{*}\left(A \bar{x}_{k}-B \bar{y}_{k}\right)\right\|^{2}}\right\}$, and $\alpha_{k}=$ $\min \left(\frac{1}{(k+1)^{3 / 2} \eta_{k}}, \frac{1}{(k+1) \zeta_{k}}, 0.94\right)$, where $\eta_{k}=\left\|x_{k}-x_{k-1}\right\|^{2}+\left\|y_{k}-y_{k-1}\right\|^{2}$,

$\zeta_{k}=\left\|x_{k}-x_{k-1}\right\|+\left\|y_{k}-y_{k-1}\right\|$. For comparison, the same random values are taken in each test for two algorithms.

Table 1: Computational results for Example 4.1 with different dimensions.

\begin{tabular}{cccccccc}
\hline & $\mathrm{J}$ & & 20 & 40 & 60 & 80 & 100 \\
\hline$(\mathrm{N}, \mathrm{M})=(50,50)$ & Algorithm 1.2 & Iter. & 720 & 4870 & 24162 & 14806 & 131045 \\
& & Sec. & 0.172 & 2.293 & 6.318 & 5.990 & 36.504 \\
$(\mathrm{~N}, \mathrm{M})=(50,50)$ & \multirow{2}{*}{ Algorithm 1.3 } & Iter. & 247 & 2509 & 7539 & 8055 & 78855 \\
& & Sec. & 0.078 & 1.264 & 2.231 & 3.526 & 24.633 \\
\hline$(\mathrm{N}, \mathrm{M})=(80,100)$ & Algorithm 1.2 & Iter. & 182 & 1668 & 3482 & 11198 & 22431 \\
& & Sec. & 0.062 & 0.484 & 1.295 & 4.602 & 11.528 \\
$(\mathrm{~N}, \mathrm{M})=(80,100)$ & \multirow{2}{*}{ Algorithm 1.3 } & Iter. & 168 & 337 & 719 & 2529 & 4575 \\
& & Sec. & 0.078 & 0.125 & 0.296 & 1.154 & 2.730 \\
\hline$(\mathrm{N}, \mathrm{M})=(200,150)$ & \multirow{2}{*}{ Algorithm 1.2 } & Iter. & 150 & 1011 & 2360 & 3669 & 5288 \\
& & Sec. & 0.047 & 0.437 & 1.357 & 2.605 & 5.288 \\
$(\mathrm{~N}, \mathrm{M})=(200,150)$ & \multirow{2}{*}{ Algorithm 1.3 } & Iter. & 139 & 244 & 329 & 707 & 876 \\
& & Sec. & 0.047 & 0.125 & 0.218 & 0.562 & 0.920 \\
\hline
\end{tabular}

From Table 1, we could observe that the inertial projection Algorithm 1.3 behaves far better than Algorithm 1.2 from the number of iterations and the cpu time. It really speeds up Algorithm 1.2.

\section{Acknowledgment}

This work was supported National Natural Science Foundation of China (No. 71602144) and Open Fund of Tianjin Key Lab for Advanced Signal Processing (No. 2016ASP-TJ01).

\section{References}

[1] F. Alvarez, Weak convergence of a relaxed and inertial hybrid projection-proximal point algorithm for maximal monotone operators in Hilbert space, SIAM J. Optim., 14 (2004), 773-782. 2.4

[2] H. Attouch, J. Bolte, P. Redont, A. Soubeyran, Alternating proximal algorithms for weakly coupled convex minimization problems, Applications to dynamical games and PDE's, J. Convex Anal., 15 (2008), 485-506. 1 
[3] H. Attouch, A. Cabot, F. Frankel, J. Peypouquet, Alternating proximal algorithms for linearly constrained variational inequalities: application to domain decomposition for PDE's, Nonlinear Anal., 74 (2011), 7455-7473. 1

[4] H. H. Bauschke, P. L. Combettes, Convex analysis and monotone operator theory in Hilbert spaces, With a foreword by Hédy Attouch, CMS Books in Mathematics/Ouvrages de Mathmatiques de la SMC, Springer, New York, (2011). 2.5

[5] A. Beck, M. Teboulle, A fast iterative shrinkage-thresholding algorithm for linear inverse problems, SIAM J. Imaging Sci., 2 (2009), 183-202. 1

[6] C. Byrne, A. Moudafi, Extensions of the CQ algorithm for the split feasibility and split equality problems (10th draft), working paper or preprint, hal-00776640, (2012). 1

[7] Y. Censor, T. Bortfeld, B. Martin, A. Trofimov, A unified approach for inversion problems in intensity-modulated radiation therapy, Phys. Med. Biol., 51 (2006), 2353-2365. 1

[8] A. Chambolle, C. Dossal, On the convergence of the iterates of the "fast iterative shrinkage/thresholding algorithm", J. Optim. Theory. Appl., 166 (2015), 968-982. 1, 3.1

[9] Q.-L. Dong, Y.-J. Cho, L.-L. Zhong, T. M. Rassias, Inertial projection and contraction algorithms for variational inequalities, J. Global Optim., 2017 (2017), 1-18. 1

[10] Q.-L. Dong, S.-N. He, Self-adaptive projection algorithms for solving the split equality problems, Fixed Point Theory, accepted. 1

[11] Q.-L. Dong, S.-N. He, J. Zhao, Solving the split equality problem without prior knowledge of operator norms, Optimization, 64 (2015), 1887-1906. 1, 4

[12] Q.-L. Dong, Y.-Y. Lu, J.-F. Yang, The extragradient algorithm with inertial effects for solving the variational inequality, Optimization, 65 (2016), 2217-2226. 1

[13] Q.-L. Dong, H.-B. Yuan, Y.-J. Cho, T. M. Rassias, Modified inertial Mann algorithm and inertial CQ-algorithm for nonexpansive mappings, Optim. Lett., 2017 (2017), 1-6, accepted. 1

[14] D. A. Lorenz, T. Pock, An inertial forward-backward algorithm for monotone inclusions, J. Math. Imaging Vision, 51 (2015), 311-325. 1

[15] A. Moudafi, A relaxed alternating CQ-algorithm for convex feasibility problems, Nonlinear Anal., 79 (2013), 117-121. 1

[16] A. Moudafi, Alternating CQ-algorithms for convex feasibility and split fixed-point problems, J. Nonlinear Convex Anal., 15 (2014), 809-818. 1, 3.1

[17] A. Moudafi, E. Al-Shemas, Simultaneous iterative methods for split equality problem, Trans. Math. Program. Appl., 1 (2013), 1-11. 1

[18] A. Moudafi, M. Oliny, Convergence of a splitting inertial proximal method for monotone operators, J. Comput. Appl. Math., 155 (2003), 447-454. 1

[19] D.-L. Tian, L.-Y. Shi, R.-D. Chen, Iterative algorithm for solving the multiple-sets split equality problem with split selfadaptive step size in Hilbert spaces, J. Inequal. Appl., 2016 (2016), 9 pages. 1 